\title{
BRAF V600E-Mutant Colorectal Cancers with Liver- Only Metastases: A Retrospective Study.
}

\section{Sahir Javed}

Centre Oscar Lambret

\section{Stéphane Benoist}

Hospital Bicetre: Hopital Bicetre

\section{Patrick Devos}

University of Lille: Universite de Lille

\section{Stéphanie Truant}

CHRU de Lille: Centre Hospitalier Universitaire de Lille

\section{Rosine Guimbaud}

CHU Toulouse: Centre Hospitalier Universitaire de Toulouse

\section{Astrid Lièvre}

CHU Rennes: Centre Hospitalier Universitaire de Rennes

\section{David Sefrioui}

CHU Rouen: Centre Hospitalier Universitaire de Rouen

\section{Romain Cohen}

Hôpital Saint-Antoine: Hopital Saint-Antoine

\section{Pascal Artru}

Jean Mermoz Private Hospital: Hopital Prive Jean Mermoz

\section{Aurélien Dupré}

Centre Léon Bérard: Centre Leon Berard

\section{Jean-Baptiste Bachet}

Hospital Pitie-Salpetriere: Hopital Universitaire Pitie Salpetriere

Christelle de la Fourchardière

Centre Léon Bérard: Centre Leon Berard

\section{Anne Ploquin}

CHRU de Lille: Centre Hospitalier Universitaire de Lille

Anthony Turpin ( $\nabla$ anthony.turpin@chru-lille.fr)

CHRU de Lille: Centre Hospitalier Universitaire de Lille https://orcid.org/0000-0002-2282-0101

\section{Research Article}

Keywords: Colorectal cancer, BRAF mutation, Hepatectomy, Drug therapy 
Posted Date: August 3rd, 2021

DOI: https://doi.org/10.21203/rs.3.rs-744993/v1

License: (c) (i) This work is licensed under a Creative Commons Attribution 4.0 International License. Read Full License 


\section{Abstract}

\section{BACKGROUND}

$B R A F$ V600E-mutant colorectal cancers (CRCs) are associated with shorter survival than BRAF wild-type tumors. Therapeutic decision-making for colorectal liver metastases (CRLM) harboring this mutation remains difficult due to the scarce literature. Our aim was to study a large cohort of BRAFV600E-mutant CRLM patients in order to identify prognostic factors associated with overall survival.

\section{METHODS}

We retrospectively identified $B R A F \mathrm{~V} 600 \mathrm{E}$-mutant CRCs diagnosed with liver-only metastases, resected or not, between April 2008 and December 2017, in 25 French centers. Clinical, molecular, pathological characteristics and treatment features were collected. Overall survival (OS) was defined as the time from CRLM diagnosis to death from any cause. Cox proportional hazard models were used for statistical analysis.

\section{RESULTS}

Among the 105 patients included, 79 (75\%) received chemotherapy, 18 (17\%) underwent upfront CRLM surgery, and 8 (8\%) received exclusive best supportive care. CRLM surgery was performed in $49(46.7 \%)$ patients. CRLM were mainly synchronous (90\%) with bilobar presentation $(61 \%)$. The median OS was 34 months (range, 28.9-67.3 months) for resected patients and 10.6 (6.7-12.5) months for unresected patients $(P<0.0001)$. Most patients received doublet chemotherapy $(72 \%)$, and $34 \%$ received a combination with bevacizumab as a first-line treatment. In multivariate analysis, primary tumor surgery (hazard ratio $(\mathrm{HR})=0.349 ; 95 \%$ confidence interval $(\mathrm{Cl}) 0.164-0.744, P=0.0064$ ) and CRLM resection $(\mathrm{HR}=0.169 ; 95 \% \mathrm{Cl} 0.082-0.348, P<0.0001)$ were associated with significantly better OS.

\section{CONCLUSIONS}

In the era of systemic cytotoxic chemotherapies, liver surgery seems to extend OS. Therefore, CRLM resectability should be assessed, regardless of mutational status.

\section{Background}

Approximately $50 \%$ of patients with colorectal cancer develop colorectal liver metastases (CRLM), and their outcomes are intimately related to CRLM resectability: the 5-year overall survival (OS) rate ranges from $30-50 \%$ after CRLM surgery, whereas it is lower than $10 \%$ for unresectable CRLM ${ }^{[1,2]}$. However, $50-$ 
$85 \%$ of patients experience relapse after CRLM resection, and the curative intent of metastasectomy is

accomplished in approximately $20 \%$ of case $^{[3-5]}$. In the era of precision medicine, efforts are aimed at a better selection of patients who might benefit from metastasectomy. Several clinical scoring systems based on clinicopathological parameters have been proposed; however, but their clinical value is still questioned ${ }^{[6,7]}$.

Colorectal cancers (CRCs) harboring BRAF V600E mutations are aggressive cancers with rapid metastatic spread that more frequently involves peritoneal and nodal invasion than liver metastases. Until recently, their management was based on limited data, mainly from subgroup analysis of randomized clinical trials. This subgroup of patients is less responsive to standard chemotherapies. In the CALGB/SWOG 80405 trial assessing the addition of the targeted agent cetuximab or bevacizumab or both to doublet chemotherapy FOLFOX or FOLFIRI, the median OS for BRAF-mutant patients remained poor compared to that of $B R A F$ wild-type patients: 13.5 months versus 30.6 months, respectively $(P<0.001)^{[8]}$. In addition, a recent meta-analysis of five randomized clinical trials demonstrated that intensive upfront chemotherapy with triplet FOLFOXIRI plus bevacizumab did not improve survival among BRAFV600E-mutant patients ${ }^{[9]}$.

This gap in survival rates has been also observed after CRLM resection in several retrospective subgroup analyses. In the latest study, the 3-year OS rates for BRAF-mutant and wild-type patients were $54 \%$ and $82.9 \%$, respectively ${ }^{[10]}$. However, these numbers must be interpreted with caution as BRAF-mutant CRCs with liver-only metastases represent a limited population, and only $5 \%$ of patients undergoing CRLM resection harbor these mutations ${ }^{[11-13]}$.

Therefore, our knowledge about BRAF-mutant patients with CRLM is currently limited to those patients undergoing resection or with extra-hepatic metastases receiving chemotherapies. In this study, we aimed to report outcomes of a large cohort of BRAF V600E-mutant patients with exclusive CRLM, resected or not, to identify potential prognostic factors.

\section{Methods}

\section{Study Population and Design}

Data from 105 patients diagnosed with liver-limited CRC metastases harboring BRAF V600E mutations between April 1, 2008 and December 31, 2017 were retrospectively collected from 25 French hospitals. Exclusion criteria were: presence of extra-hepatic metastases, date of CRLM diagnosis not available, and follow-up less than 12 months. Data from the majority of patients who underwent CRLM resection came from databases of the following four French scientific groups: Fédération de Recherche et Chirurgie (FRENCH), Association de Chirurgie Hépato-Bilio-Pancréatique et Transplantation (ACHBT), Association des Gastro-Enterologues Oncologues (AGEO), and the PRODIGE group (Partenariat de Recherche en Oncologie DIGEstive). BRAF V600E mutated-patients were identified from molecular biology platforms and each case was screened in order to identify and include patients with liver-only disease. Patients with synchronous extra-hepatic resectable disease metastases were excluded. The study was conducted 
according to the ethical standards in line with the French regulation. French Data Protection Authority (CNIL agreement $n^{\circ}$ DEC18-409 (2018_01)) provided a waiver of informed consent for this retrospective study and permitted the publication of anonymized data.

$B R A F$ and RAS mutational statuses were determined from either primary CRC samples or CRLM tissuesas several studies have demonstrated a high molecular concordance between primary CRC and liver metastases ${ }^{[14,15]}$-using PCR or next-generation sequencing.

The following clinical, molecular, and pathological characteristics were collected at baseline: age at CRLM diagnosis, sex, KRAS and NRAS mutations, mismatch repair (MMR) status, primary tumor site, surgery of primary tumor, tumor and nodal stages according to the American Joint Committee on Cancer, synchronous ( $<6$ months) versus metachronous CRLM diagnosis, CRLM distribution and number, and initial resectability status. In addition, treatment features (CRLM surgery and systemic therapies) and survival were assembled.

MMR status was assessed by both immunohistochemical analysis of microsatellite instability-high (MSIH) defined by loss of MLH1, MSH2, MSH6, or PMS2 expression and PCR. Right-sided tumors were defined as arising from the caecum to the transverse colon and left-sided tumors as arising from the splenic flexure to the rectosigmoid junction.

\section{Treatment Features and Definitions}

The treatment decision for each patient was made during multidisciplinary meetings in each institution. According to the CRLM resectability status and performance status, patients received preoperative chemotherapy, upfront liver surgery, palliative chemotherapy, or best supportive care. Patients were then followed-up every 2-3 months through physical examination, biological tests, and computed tomography scan. OS was defined as the time from CRLM diagnosis to the time of death or the date of last follow-up. Post-operative OS was defined as the time from CRLM resection.

\section{Statistical Analysis}

For descriptive analysis, quantitative parameters are presented as median and quartiles and qualitative parameters as percentages. CRLM resected and unresected groups were compared using the $\chi 2$ or Fisher's exact test, as appropriate. Survival rates were estimated by the Kaplan-Meier method and were compared using the log-rank test. After univariate analysis, significant variables $(P<0.2)$ with sufficient data available (70 out of 105 patients) were integrated in a backward selection procedure to keep factors significant in the final multivariate Cox model. Boostrap methods were also used. The variables of interest were: age, gender, primary tumor site, primary tumor surgery, synchronous CRLM, CRLM number, CRLM distribution, resectability status, metastasectomy, and the use of first-line chemotherapy and targeted therapies. All reported $P$ values are two-sided, and $P<0.05$ was considered statistically significant. Statistical analyses were performed using SAS V9.4 (Cary, NC, USA).

\section{Results}




\section{Patient Characteristics}

We identified 105 patients with BRAF V600E-mutant CRLM diagnosed between April 2008 and December 2017. The median age at CRLM diagnosis was 67 years. CRLM were mainly synchronous (90\%) with bilobar presentation (61\%). One patient harbored co-KRAS mutation. MMR status was available for 69 patients (66\%): 21 patients (30\%) were identified with an MSI-H phenotype. Clinical, molecular, and pathological characteristics are summarized in Table 1. 
Table 1

Patient characteristics according to CRLM status (resected or unresected)

\begin{tabular}{|c|c|c|c|c|}
\hline Variables & $\begin{array}{l}\text { Total } \\
(n=105)\end{array}$ & $\begin{array}{l}\text { Resected CRLM } \\
(n=49)\end{array}$ & $\begin{array}{l}\text { Unresected CRLM } \\
(n=56)\end{array}$ & $P$ value \\
\hline \multicolumn{5}{|l|}{ Gender } \\
\hline Male & $51(49 \%)$ & $20(41 \%)$ & $31(55 \%)$ & \multirow[t]{2}{*}{0.1369} \\
\hline Female & $54(51 \%)$ & $29(59 \%)$ & $25(45 \%)$ & \\
\hline \multicolumn{5}{|l|}{ Age } \\
\hline$\leq 65$ years & $42(40 \%)$ & $20(41 \%)$ & $22(39 \%)$ & \multirow[t]{2}{*}{0.8731} \\
\hline$>65$ years & $63(60 \%)$ & $29(59 \%)$ & $34(61 \%)$ & \\
\hline \multicolumn{5}{|c|}{ Primary tumor site } \\
\hline Right-sided & $56(55 \%)$ & $26(54 \%)$ & $30(56 \%)$ & \multirow[t]{4}{*}{0.0911} \\
\hline Left-sided & $28(27 \%)$ & $17(36 \%)$ & $11(20 \%)$ & \\
\hline Rectum & $18(18 \%)$ & $5(10 \%)$ & $13(24 \%)$ & \\
\hline Missing data & 3 & 1 & 2 & \\
\hline \multicolumn{5}{|c|}{ Primary tumor surgery } \\
\hline Yes & $79(75 \%)$ & $49(100 \%)$ & $30(54 \%)$ & \multirow[t]{2}{*}{$<0.0001$} \\
\hline No & $26(25 \%)$ & 0 & $26(46 \%)$ & \\
\hline \multicolumn{5}{|l|}{ T stage } \\
\hline $\mathrm{T} 1-\mathrm{T} 2$ & $4(5 \%)$ & $4(8 \%)$ & 0 & \multirow[t]{3}{*}{0.2909} \\
\hline T3-T4 & $73(95 \%)$ & $44(92 \%)$ & $29(100 \%)$ & \\
\hline Missing data & 28 & 1 & 27 & \\
\hline \multicolumn{5}{|l|}{$\mathrm{N}$ stage } \\
\hline NO & $9(12 \%)$ & $7(15 \%)$ & $2(7 \%)$ & \multirow[t]{3}{*}{0.4699} \\
\hline $\mathrm{N} 1-\mathrm{N} 2$ & $68(88 \%)$ & $41(85 \%)$ & $27(93 \%)$ & \\
\hline Missing data & 28 & 1 & 27 & \\
\hline \multicolumn{5}{|c|}{ CRLM time of diagnosis } \\
\hline Synchronous & $94(90 \%)$ & $41(84 \%)$ & 53 (95\%) & \multirow[t]{2}{*}{0.0671} \\
\hline Metachronous & $11(10 \%)$ & $8(16 \%)$ & $3(5 \%)$ & \\
\hline
\end{tabular}




\begin{tabular}{|c|c|c|c|c|}
\hline Variables & $\begin{array}{l}\text { Total } \\
(n=105)\end{array}$ & $\begin{array}{l}\text { Resected CRLM } \\
(n=49)\end{array}$ & $\begin{array}{l}\text { Unresected CRLM } \\
(n=56)\end{array}$ & $P$ value \\
\hline Unilobar & $33(39 \%)$ & $21(57 \%)$ & $12(26 \%)$ & 0.0036 \\
\hline Bilobar & $51(61 \%)$ & $16(43 \%)$ & 35 (74\%) & \\
\hline Missing data & 21 & 12 & 9 & \\
\hline \multicolumn{5}{|c|}{ Number of CRLM } \\
\hline$<10$ & $56(70 \%)$ & 32 (89\%) & $24(55 \%)$ & $<0.0001$ \\
\hline$\geq 10$ & $24(30 \%)$ & $4(11 \%)$ & $20(45 \%)$ & \\
\hline Missing data & 25 & 13 & 12 & \\
\hline \multicolumn{5}{|c|}{ Initial resectability } \\
\hline Yes & $43(52 \%)$ & $32(86 \%)$ & $11(24 \%)$ & $<0.0001$ \\
\hline No & $39(48 \%)$ & $5(14 \%)$ & $34(76 \%)$ & \\
\hline Missing data & 23 & 12 & 11 & \\
\hline \multicolumn{5}{|l|}{$R A S$ status } \\
\hline Wild-type & 103 (99\%) & 47 (98\%) & $56(100 \%)$ & 0.4615 \\
\hline Mutant & $1(1 \%)$ & $1(2 \%)$ & 0 & \\
\hline Missing data & & 1 & - & \\
\hline \multicolumn{5}{|l|}{ MSI-H } \\
\hline Yes & $21(30 \%)$ & $14(29 \%)$ & $7(33 \%)$ & 0.7293 \\
\hline No & $48(70 \%)$ & $34(71 \%)$ & $14(67 \%)$ & \\
\hline Missing data & 36 & 1 & 35 & \\
\hline
\end{tabular}

\section{Treatment Features}

The flow chart in Fig. 1 describes the treatments administrated. Forty-nine out of 105 patients (47\%) underwent CRLM resection, of which 31 (63\%) after chemotherapy. Major liver resection ( $\geq 3$ segments) was performed in $38 \%$ of cases (14/37), two-stage liver resection in $24 \%$ of cases $(9 / 37)$, and preoperative portal vein embolization in $11 \%$ of cases (4/37). Radiofrequency ablation was combined with liver surgery in $24 \%$ of patients (9/37). R1 parenchymal resections were present in 4 out of 36 cases (11\%). Sixty-five percent received adjuvant chemotherapy.

Cytotoxic doublet chemotherapies (FOLFOX or FOLFIRI) represented the main first-line treatment (72\%), followed by triplet chemotherapy (FOLFIRINOX) for 12 patients (15\%). Twenty-seven patients (34\%) received bevacizumab in combination with chemotherapy as the first-line treatment. 
Among patients treated exclusively with chemotherapy $(n=48), 63 \%$ received a second line $(n=30)$ and $35 \%$ received a third line $(n=17)$. From the second line onward, targeted therapies were more frequently used. In total, 24 patients (50\%) received concomitantly or successively the following cytotoxic drugs: fluoropyrimidine, oxaliplatin, and irinotecan. Of note, $54 \%$ of patients with unresected CRLM underwent primary tumor surgery (Table 1).

Finally, 10 out of 105 patients (10\%) participated in clinical trials, four of which involved immune checkpoint inhibitors (ICls) or targeted therapies.

\section{Survival Analysis}

The median OS was 16.2 months (95\% confidence interval (CI): 13.2-20.7), with a 1-year OS rate of $65 \%$ and a 3-year rate of $16 \%$ (Fig. 2). In univariate analysis, the following six factors were associated with longer survival: CRLM resection, primary tumor surgery, CRLM less $<10$, initially resectable CRLM, unilobar distribution and right and left-sided primary tumors (Table 2).

Table 2

Univariate and multivariate Cox analysis of prognostic factors for OS $(n=70)$. All other parameters (sex, age, etc.) are not significant at $P=0.20$

\begin{tabular}{|lllll|}
\hline & \multicolumn{3}{c}{ Univariate analysis } & \multicolumn{2}{c|}{ Multivariate analysis } & \\
\cline { 2 - 5 } & HR [95\% Cl] & Pvalue & HR [95\% Cl] & P value \\
\hline CRLM resection & $0.131[0.068-0.253]$ & $<0.0001$ & $0.169[0.082-0.348]$ & $<0.0001$ \\
\hline Primary tumor resection & $0.135[0.065-0.28]$ & $<0.0001$ & $0.349[0.164-0.744]$ & 0.0064 \\
\hline CRLM $\geq 10$ & $3.612[1.973-6.613]$ & $<0.0001$ & - & NS \\
\hline Non-resectable CRLM & $2.931[1.632-5.262]$ & 0.0003 & - & NS \\
\hline Bilobar CRLM & $1.697[0.931-3.092]$ & 0.0842 & - & NS \\
\hline Rectum primary site & $1.792[0.902-3.561]$ & 0.0957 & - & NS \\
\hline CRLM: colorectal liver metastases, NS: not significant & & \\
\hline
\end{tabular}

In multivariate analysis, primary tumor surgery (hazard ratio $(\mathrm{HR})=0.349 ; 95 \% \mathrm{Cl} 0.164-0.744, P=$ $0.0064)$ and $C R L M$ resection $(H R=0.169 ; 95 \% \mathrm{Cl} 0.082-0.348, P<0.0001)$ were associated with significantly better OS.

CRLM resection was associated with a significantly longer OS, with a median of 34 months (range, 28.967.3 months) versus 10.6 (6.7-12.5) months for unresected patients, $P<0.0001$ (Fig. 3). Patients who received preoperative chemotherapy had a median OS of 34 months (28.9-non-evaluable) versus 33 months $(19.6-$ non-evaluable) for patients resected upfront $(P=0.3402)$. The median post-operative OS was $28(19.8-53.5)$ months. 
Primary tumor surgery was associated with a significantly longer OS, with a median of 23.7 (16.6-33) months versus $6.4(2.9-11.1)$ months for unresected patients $(P<0.0001)$. The benefit of primary tumor surgery remained statistically significant in the unresected CRLM group $(n=30)$, with a median OS of 12.9 (9.4-16.1) months versus 6.4 (2.9-11.1) months in the unresected group (primary tumor and CRLM, $\mathrm{n}=26)(P=0.0002)$ (Fig. 4).

Exclusive chemotherapy treatment conferred a median OS of 11.5 months (7.1-13.2).

\section{Comparison of Resected and Non-Resected CRLM groups}

Clinical and pathological characteristics according to CRLM treatment status are summarized in Table 1. Patients with resected CRLM $(\mathrm{n}=49)$ were significantly more likely to present less than 10 metastases $(P$ $<0.0001)$ with unilobar distribution $(P=0.0036)$ and initially resectable $(P<0.0001)$. Considering the missing data about MSI-H status, no significant difference between the two groups was observed $(P=$ 0.7293). Concerning the radiologic responses, among the patients treated exclusively with chemotherapy, $29 \%(14 / 48)$ had an objective response, $27 \%$ (13/48) had stable disease, and 44\% (21/48) were progressive. Data were available for 25 patients who received preoperative chemotherapy, with a stable disease rate of $28 \%(7 / 25)$, objective response rate of $64 \%(16 / 25)$, and progressive disease rate of $8 \%$ $(2 / 25)$.

\section{Discussion}

Data on patients with BRAF V600E-mutant CRC and liver-only metastases are scarce. This was the largest dedicated cohort $(n=105$, regardless of treatments received) study to date, bringing an additional support that their resection is beneficial.

The profit of liver resection is in line with findings in two recent retrospective studies. Johnson et al. showed that among 52 patients with BRAF V600E metastatic CRC, the median OS was significantly prolonged when liver resection with curative intent was performed: 29.1 versus 22.7 months, HR 0.33, $95 \% \mathrm{Cl}: 0.12-0.78, P=0.01^{[16]}$. In the second study, 43 out of 282 patients underwent surgery, with a median OS of 47.4 months versus 19.5 months for those who had no metastasectomy (HR $0.469,95 \% \mathrm{Cl}$ : $0.288-0.765 ; P=0.0024)^{[17]}$. In addition, a recent case-matched study demonstrated that $B R A F$ mutations did not increase the risk of relapse after CRLM surgery compared to $B R A F$ wild-type disease (HR 1.16, 95\% Cl 0.72-1.85; $P=0.547)^{[10]}$. The high proportion of patients undergoing resection in our cohort should reflect the fact that the assessment of mutational status was probably not performed in patients with poor prognosis.

To allow comparison with unresected patients, we defined OS as the time from CRLM diagnosis, whereas previous studies have reported OS from the date of liver surgery. Nonetheless, the median post-operative OS starting from the date of surgery in our cohort ( 28 months) was in line with those in previous studies: 
from 22.6 months reported by Schirripa et al. $(n=12)$ to 47.4 months reported by De la Fouchardière et al. $(n=35)^{[17-20]}$. OS results remain lower than the previous publication from Bachet et al. with a median OS of 52.7 months $(\mathrm{n}=66)$. The exclusion of non-V600E BRAF mutated-patients in our study may explain this difference ${ }^{[10]}$. Relapse free survival and progression free survival were not included in our study as the definitions would differ for resected and unresected groups.

The positive results of primary tumor resection in the unresected CRLM group were surprising and should be interpreted with caution due to the small number of patients involved (30 versus 26 ). Indeed, a recent study showed that primary tumor resection followed by chemotherapy was not superior to chemotherapy alone (HR 1.10 [0.76-1.59], one-sided $P=0.69$ ). The trial was terminated early for futility reason ${ }^{[21]}$.

In our cohort, a small proportion of patients received an upfront triplet regimen with or without bevacizumab $(12 / 79,15 \%)$, with a median OS of 16.6 months (6.7-not reached). Of note, the majority of the cohort presented a good performance status (84\% in the group treated exclusively with chemotherapy). An intensive regimen has been assumed beneficial in BRAF-mutant patients with unresectable liver-limited disease to date, based on a pooled analysis of a small number of patients $(\mathrm{n}=$ $20)^{[22]}$. However, a recent meta-analysis of five randomized trials comparing FOLFOXIRI plus bevacizumab with doublet plus bevacizumab in 105 BRAF V600E-mutant patients showed no increased benefit in the intensive therapeutic arm ${ }^{[9]}$.

Among the patients treated with chemotherapy only, $63 \%$ received second-line treatment; this rate is superior to those reported in the COIN trial (33\%) (23) and in a matched case-control study (51\%) ${ }^{[24]}$. Beyond the second line, candidates for treatment decreased dramatically to $35 \%$, and it is important to pinpoint that only $50 \%$ of patients received the three major cytotoxic drugs: 5 -FU/leucovorin, oxaliplatin, and irinotecan. Our study showed that even if BRAF-mutant metastatic disease is confined to one organ, the prognosis remains poor when the patient is treated with chemotherapy only.

Our study population mostly received standard chemotherapies, not new practice-changing therapies. Recently, mitogen-activated protein kinase pathway-targeted therapies have demonstrated better efficacy. In the BEACON trial, the combination of encorafenib, a BRAF inhibitor, and cetuximab (anti-EGFR) with or without binimetinib, a MEK inhibitor, was associated with a significantly longer OS than standard chemotherapy after at least one prior line in a large cohort of patients with BRAF V600E mutations ${ }^{[25]}$. The same regimen as a first-line is currently under investigation in a phase II trial (ANCHOR-CRC) ${ }^{[26]}$.

ICls represent another therapeutic option, especially for MSI-H mCRC. The phase III KEYNOTE-177 study demonstrated that first-line pembrolizumab was associated with significant progression-free survival improvement over chemotherapy in MSI-H mCRC (median progression-free survival of 16.5 versus 8.2 months, $\mathrm{HR}=0.60 ; 95 \% \mathrm{Cl} 0.45-0.80, P=0.0002$ ). The benefit of pembrolizumab was consistent in the $B R A F$ V600E-mutant subgroup ${ }^{[27]}$. In our cohort, very few patients received ICl after chemotherapy failure $(n=3)$, explained by the period of inclusion. MSI-H status was not reliable for any conclusions due to 
insufficient data. In a recent study, MSI-H status was associated with significantly longer OS in a BRAFmutant mCRC population $(n=194)$ treated with standard chemotherapies ${ }^{[17]}$.

The major weakness of our study is related to the differences between the resected and unresected CRLM groups with the bias of less aggressive disease in the resected group. However, this should be counterbalanced by increased liver surgery ability and moreover, the initial resectability or unresectability status might have been subject to variability between the centers. The reasons for non-resectability were not specified, and patients could be considered unresectable solely based on the presence of $B R A F$ mutations.

The missing data in our cohort represent an important limitation, and some known prognostic factors, such as MSI status, were not included in the statistical analysis. Therefore, a case-matched study (resected and unresected CRLM) was not feasible. A prospective study with current therapeutic strategies (ICl for MSI-H and anti-BRAF plus anti-MEK for non-MSI-H) should be considered.

With all the limitations of a retrospective study, this was conducted in the largest cohort of BRAF V600E mutant patients with CRLM reported to date. A subgroup difficult to look at given its rarity, also prospective studies would be difficult to realize.

\section{Conclusions}

As long as systemic targeted therapies and immunotherapies are under development, liver resection seems to improve survival time than chemotherapy alone. Therefore, colorectal liver metastases with $B R A F \mathrm{~V} 600 \mathrm{E}$ mutations should not be excluded from liver surgery when considered and/or rendered resectable.

\section{Abbreviations}

$\mathrm{Cl}$

Confidence interval

CRC

Colorectal cancer

CRLM

Colorectal liver metastases

HR

Hazard ratio

ICI

Immune checkpoint inhibitor

\section{MMR}

Mismatch repair

MSI-H 
Microsatellite instability

OS

Overall survival

PCR

Polymerase chain reaction

\section{Declarations}

- Ethics approval and consent to participate:

- The study was conducted according to the ethical standards in line with the French regulation. French Data Protection Authority (CNIL agreement n DEC18-409 (2018_01)) provided a waiver of informed consent for this retrospective study and permitted the publication of anonymized data.

- Non-deceased patients at the time of the study were informed and declared non-opposition to medical data collection and the data were anonymously analyzed.

\section{- Consent for publication:}

- French Data Protection Authority (CNIL agreement n DEC18-409 (2018_01)) provided a waiver of informed consent for this retrospective study and permitted the publication of anonymized data.

- Availability of data and materials:

- The datasets used and/or analysed during the current study are available from the corresponding author on reasonable request.

- Competing interests :

- The authors declare that they have no competing interests

- Funding: none

\section{- Authors' contributions:}

- SJ, AP and AT designed the study. SJ, SB and CD collected the patient's clinical data. PD performed data processing and statistical analysis. SJ analyzed the data and wrote the paper under supervision of AP and AT. All authors revised and approved the final version of the manuscript.

\section{- Acknowledgements:}

- We would like to thank Julien Edeline, Farid El Hajbi, Marie-Pierre Galais, Claire Giraud, Vincent Hautefeuille, Olivier Romano, who helped to get access to patients'clinical data from their hospital.

- We would like to thank Editage (www.editage.com) for English language editing

\section{References}


1. Adam R, Delvart V, Pascal G, Valeanu A, Castaing D, Azoulay D, et al. Rescue surgery for unresectable colorectal liver metastases downstaged by chemotherapy: a model to predict long-term survival. Ann Surg. 2004;240(4):644-57.

2. Nordlinger B, Sorbye H, Glimelius B, Poston GJ, Schlag PM, Rougier P, et al. Perioperative FOLFOX4 chemotherapy and surgery versus surgery alone for resectable liver metastases from colorectal cancer (EORTC 40983): long-term results of a randomised, controlled, phase 3 trial. Lancet Oncol. 2013;14(12):1208-15.

3. Viganò L, Ferrero A, Lo Tesoriere R, Capussotti L. Liver Surgery for Colorectal Metastases: Results after 10 Years of Follow-Up. Long-Term Survivors, Late Recurrences, and Prognostic Role of Morbidity. Ann Surg Oncol. 2008;15(9):2458-64.

4. de Jong MC, Pulitano C, Ribero D, Strub J, Mentha G, Schulick RD, et al. Rates and Patterns of Recurrence Following Curative Intent Surgery for Colorectal Liver Metastasis: An International MultiInstitutional Analysis of 1669 Patients. Trans Meet Am Surg Assoc. 2009;127:84-92.

5. Pulitanò C, Castillo F, Aldrighetti L, Bodingbauer M, Parks RW, Ferla G, et al. What defines « cure » after liver resection for colorectal metastases? Results after 10 years of follow-up. HPB. 2010;12(4):244-9.

6. Nordlinger B, Guiguet M, Vaillant JC, Balladur P, Boudjema K, Bachellier P, et al. Surgical resection of colorectal carcinoma metastases to the liver. A prognostic scoring system to improve case selection, based on 1568 patients. Association Française de Chirurgie Cancer. 1996;77(7):1254-62.

7. Fong Y, Fortner J, Sun RL, Brennan MF, Blumgart LH. Clinical score for predicting recurrence after hepatic resection for metastatic colorectal cancer: analysis of 1001 consecutive cases. Ann Surg. 1999;230(3):309-18. discussion 318-321.

8. Innocenti F, Ou F-S, Qu X, Zemla TJ, Niedzwiecki D, Tam R, et al. Mutational Analysis of Patients With Colorectal Cancer in CALGB/SWOG 80405 Identifies New Roles of Microsatellite Instability and Tumor Mutational Burden for Patient Outcome. J Clin Oncol Off J Am Soc Clin Oncol. 2019;37(14):1217-27.

9. Cremolini C, Antoniotti C, Stein A, Bendell JC, Gruenberger T, Masi G, et al. FOLFOXIRI/bevacizumab (bev) versus doublets/bev as initial therapy of unresectable metastatic colorectal cancer (mCRC): A meta-analysis of individual patient data (IPD) from five randomized trials. J Clin Oncol. 2020;38(15_suppl):4015-5.

10. Bachet J-B, Moreno-Lopez N, Vigano L, Marchese U, Gelli M, Raoux L, et al. BRAF mutation is not associated with an increased risk of recurrence in patients undergoing resection of colorectal liver metastases. Br J Surg. 2019;106(9):1237-47.

11. Teng H-W, Huang Y-C, Lin J-K, Chen W-S, Lin T-C, Jiang J-K, et al. BRAF mutation is a prognostic biomarker for colorectal liver metastasectomy. J Surg Oncol. 2012;106(2):123-9.

12. Passiglia F, Bronte G, Bazan V, Galvano A, Vincenzi B, Russo A. Can KRAS and BRAF mutations limit the benefit of liver resection in metastatic colorectal cancer patients? A systematic review and metaanalysis. Crit Rev Oncol Hematol. 2016;99:150-7. 
13. Tosi F, Magni E, Amatu A, Mauri G, Bencardino K, Truini M, et al. Effect of KRAS and BRAF Mutations on Survival of Metastatic Colorectal Cancer After Liver Resection: A Systematic Review and MetaAnalysis. Clin Colorectal Cancer. 2017;16(3):e153-63.

14. Kim K-P, Kim J-E, Hong YS, Ahn S-M, Chun SM, Hong S-M, et al. Paired Primary and Metastatic Tumor Analysis of Somatic Mutations in Synchronous and Metachronous Colorectal Cancer. Cancer Res Treat Off J Korean Cancer Assoc. 2017;49(1):161-7.

15. Bhullar DS, Barriuso J, Mullamitha S, Saunders MP, O’Dwyer ST, Aziz O. Biomarker concordance between primary colorectal cancer and its metastases. EBioMedicine. 2019;40:363-74.

16. Johnson B, Jin Z, Truty MJ, Smoot RL, Nagorney DM, Kendrick ML, et al. Impact of Metastasectomy in the Multimodality Approach for BRAF V600E Metastatic Colorectal Cancer: The Mayo Clinic Experience. Oncologist. 2018;23(1):128-34.

17. de la Fouchardière $C$, Cohen $R$, Malka $D$, Guimbaud $R$, Bourien $H$, Lièvre $A$, et al. Characteristics of BRAF V600E Mutant, Deficient Mismatch Repair/Proficient Mismatch Repair, Metastatic Colorectal Cancer: A Multicenter Series of 287 Patients. The Oncologist. 2019.

18. Schirripa M, Bergamo F, Cremolini C, Casagrande M, Lonardi S, Aprile G, et al. BRAF and RAS mutations as prognostic factors in metastatic colorectal cancer patients undergoing liver resection. Br J Cancer. 2015;112(12):1921-8.

19. Margonis GA, Buettner S, Andreatos N, Kim Y, Wagner D, Sasaki K, et al. Association of BRAF Mutations With Survival and Recurrence in Surgically Treated Patients With Metastatic Colorectal Liver Cancer. JAMA Surg. 2018;153(7):e180996.

20. Gagnière J, Dupré A, Gholami SS, Pezet D, Boerner T, Gönen M, et al. Is Hepatectomy Justified for BRAF Mutant Colorectal Liver Metastases?: A Multi-institutional Analysis of 1497 Patients. Ann Surg. 2018.

21. Kanemitsu Y, Shitara K, Mizusawa J, Hamaguchi T, Shida D, Komori K, et al. A randomized phase III trial comparing primary tumor resection plus chemotherapy with chemotherapy alone in incurable stage IV colorectal cancer: JCOG1007 study (iPACS). J Clin Oncol. 2020;38(4_suppl):7-7.

22. Cremolini C, Casagrande M, Loupakis F, Aprile G, Bergamo F, Masi G, et al. Efficacy of FOLFOXIRI plus bevacizumab in liver-limited metastatic colorectal cancer: A pooled analysis of clinical studies by Gruppo Oncologico del Nord Ovest. Eur J Cancer. 2017;73:74-84.

23. Seligmann JF, Fisher D, Smith CG, Richman SD, Elliott F, Brown S, et al. Investigating the poor outcomes of BRAF-mutant advanced colorectal cancer: analysis from 2530 patients in randomised clinical trials. Ann Oncol Off J Eur Soc Med Oncol. 2017;28(3):562-8.

24. Kayhanian H, Goode E, Sclafani F, Ang JE, Gerlinger M, Gonzalez de Castro D, et al. Treatment and Survival Outcome of BRAF-Mutated Metastatic Colorectal Cancer: A Retrospective Matched CaseControl Study. Clin Colorectal Cancer. 2018;17(1):e69-76.

25. Kopetz S, Grothey A, Yaeger R, Van Cutsem E, Desai J, Yoshino T, et al. Encorafenib, Binimetinib, and Cetuximab in BRAF V600E-Mutated Colorectal Cancer. N Engl J Med. 2019;381(17):1632-43. 
26. Grothey A, Yaeger R, Paez D, Tabernero J, Taïeb J, Yoshino T, et al. ANCHOR CRC: a phase 2, openlabel, single arm, multicenter study of encorafenib (ENCO), binimetinib (BINI), plus cetuximab (CETUX) in patients with previously untreated BRAF V600E-mutant metastatic colorectal cancer (mCRC). Ann Oncol. 2019;30:iv109.

27. Andre T, Shiu K-K, Kim TW, Jensen BV, Jensen LH, Punt CJA, et al. Pembrolizumab versus chemotherapy for microsatellite instability-high/mismatch repair deficient metastatic colorectal cancer: The phase 3 KEYNOTE-177 Study. J Clin Oncol. 2020;38(18_suppl):LBA4-4.

\section{Figures}




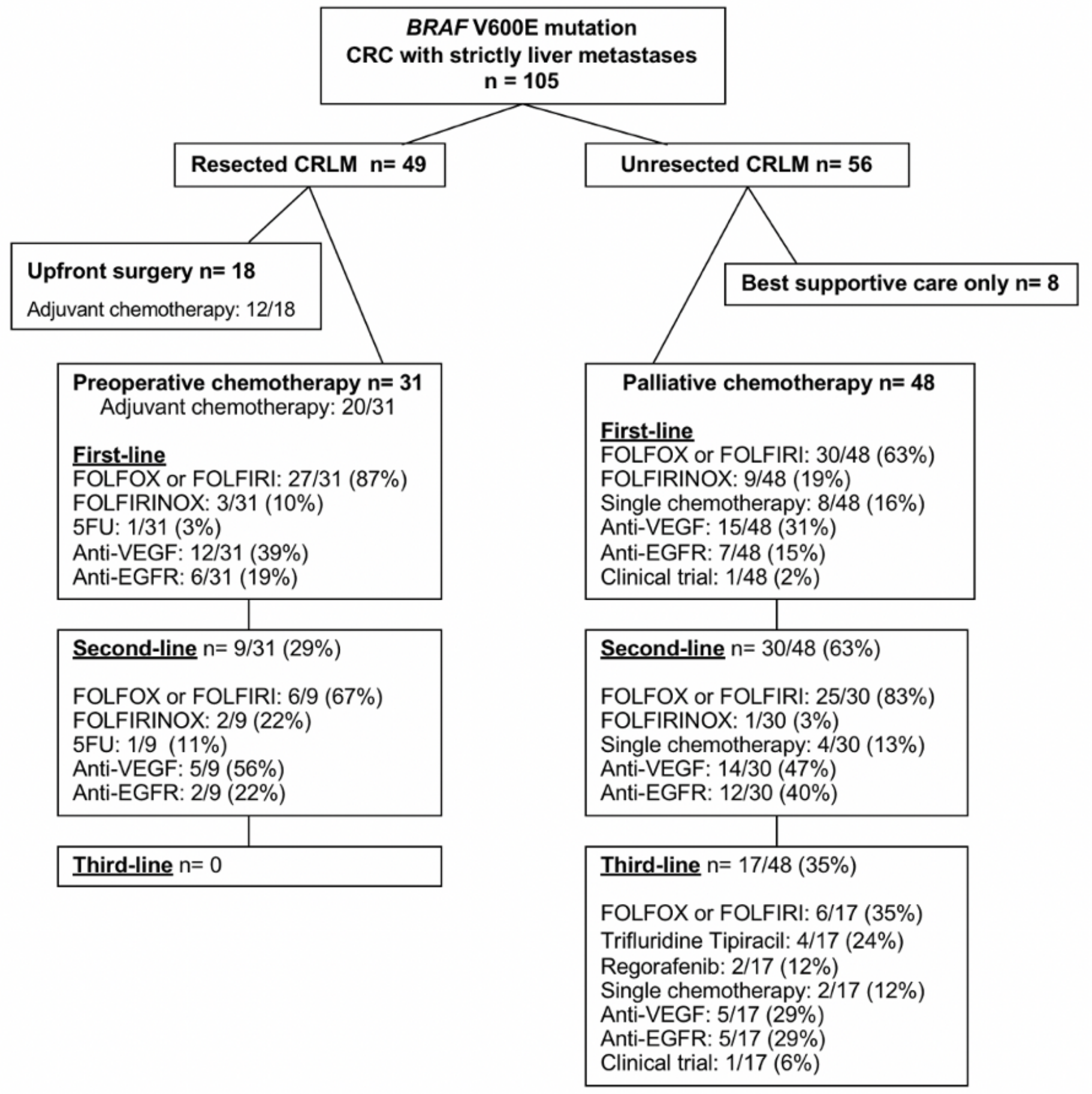

Figure 1

Flow chart of included patients $(n=105)$ 


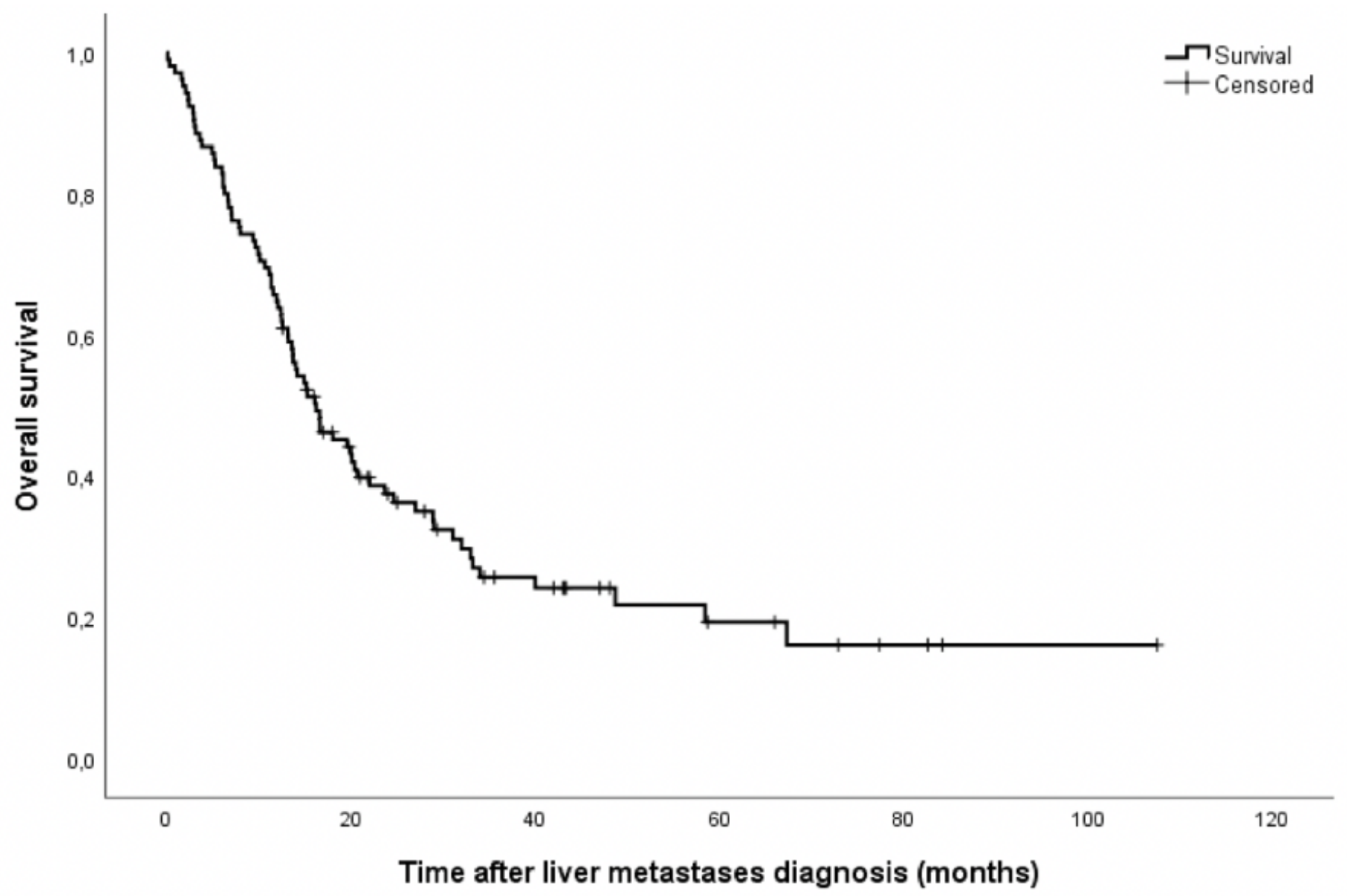

Figure 2

Overall survival for BRAF-mutant colorectal cancer with exclusive CRLM $(n=105)$, median OS $=16.2$ months 


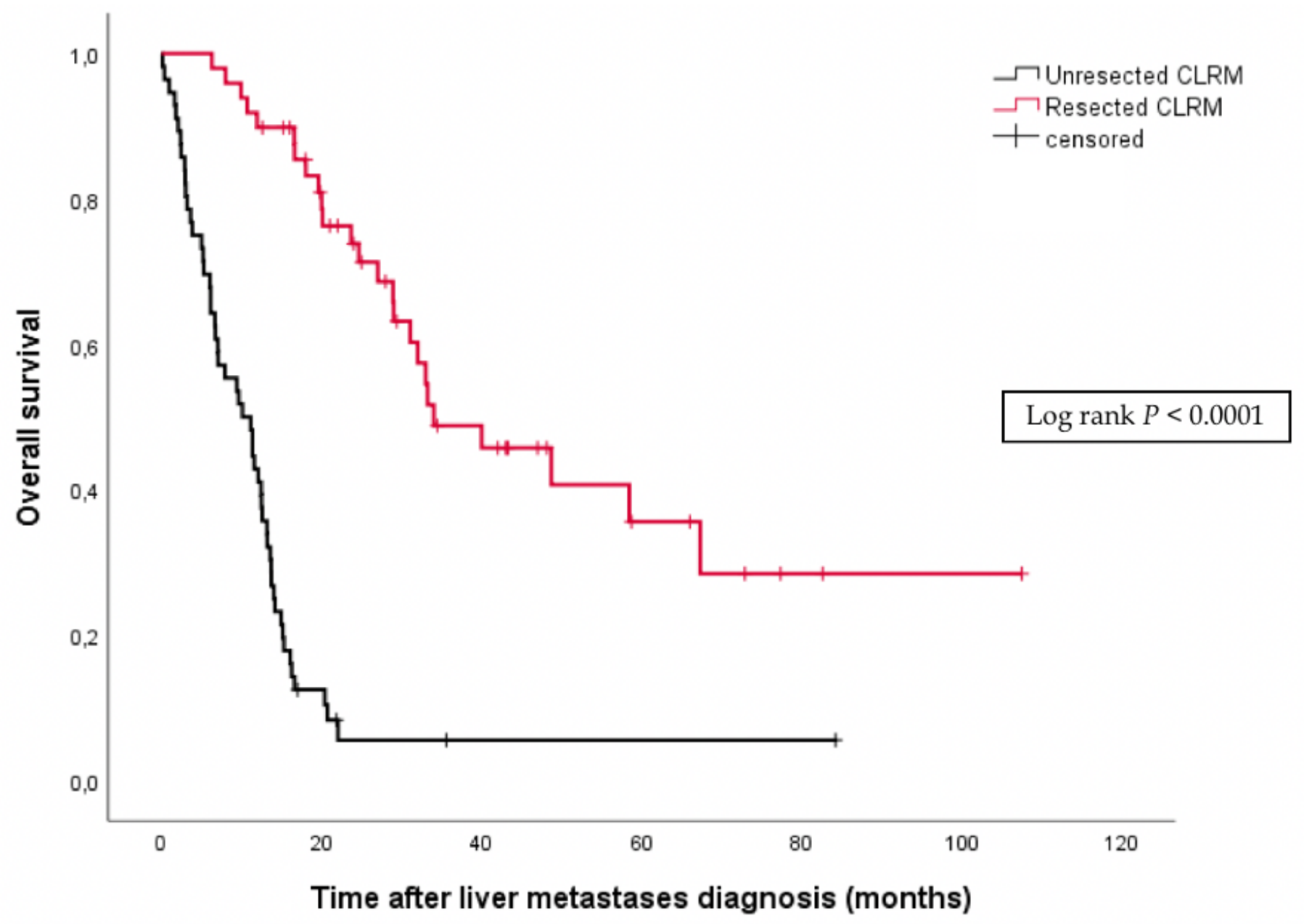

Figure 3

Overall survival according to CRLM resection status 


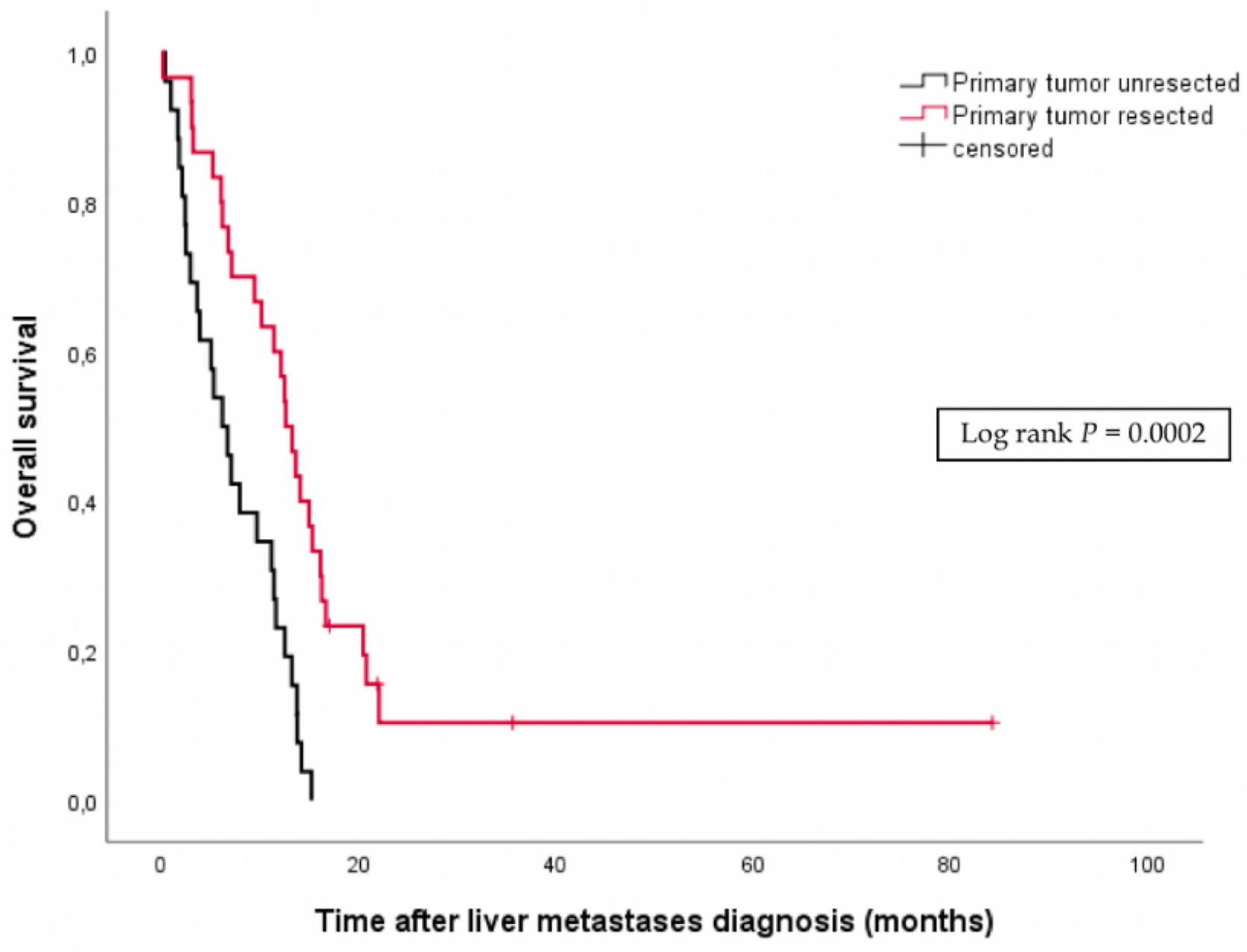

Figure 4

Overall survival according to primary tumor resection status among unresected CRLM group $(n=56)$ 